\title{
HIGH ORDER TVD SCHEME FOR HYPERBOLIC CONSERVATION LAWS
}

\author{
Amr H. Abdalla ${ }^{1}$, Anas A. M. Arafa ${ }^{2}$, Ibrahim SH I.Osman ${ }^{2, *}$ \\ ${ }^{1}$ Physics and Mathematics Department, Faculty of Engineering, Port Said University, Egypt \\ ${ }^{2}$ Mathematics Department and Computer Science, Faculty of Science, Port Said University, Egypt \\ *Corresponding author: ibrahimosman2025@gmail.com
}

\begin{abstract}
In this article, a new third order finite difference scheme for solving initial value problems for conservation laws is introduced. The advantages of this scheme are: it is a third order accuracy in space and time, is simple to implement, it has the lowest order of dissipation which reduces the oscillations generated by the numerical methods. The stability of the new scheme is proved for initial boundary value problems for linear and nonlinscalar problems. Also, the new scheme is reformulated to be total variation diminishing (TVD) i.e., oscillations free. For the nonlinear systems of equations, the extension of the new scheme is presented. Many numerical examples are presented and compared with the exact solutions and other methods.
\end{abstract}

\section{Keywords}

Conservationlaws, difference schemes, TVD schemes, Euler equations

\section{INTRODUCTION}

Since sixthy years, a substantial and productive effort to develop accurate numerical methods for hyperbolic conservation laws has been done. We are concerned with improved high order finite difference schemes. In fact, there are two types of difference methods, dissipative and dispersive schemes. For examples, leap frog scheme is strictly dispersive (non-dissipative) while the Lax Wendroff (L-W) scheme is dissipative scheme. In fact, the non-dissipative scheme conserves the $\mathrm{L}_{2}$ norms for linear equations. This reflects a property of the original differential equations. In fact, for many applications it is inecessary to minimize the energy loss. But for other applications it is better to damp the energy in the high frequencies, this can be done by using dissipative schemes. The dispersive schemes introduce the spurious oscillations behind the waves. These effects are less noticeable in dissipative schemes since the modes with greatest 
phase error are those which are mostly heavily damped. For this reason, it is better to introduce dissipative mechanisms into dispersive difference schemes. Turkel [4] introduced a composite scheme which is a threestep method. Here we introduced another method combining the diffusion scheme (dissipative) and leap frog scheme (dispersive). The resulting method and its stability properties are proved for initial and initial boundary value problems. The new scheme has the following advantages: simplicity, third order accuracy and that it has the lowest order of dissipation. To see the advantages of the scheme, comparisons with the leap frog scheme and the third order scheme presented in[5,3] are carried out. From the numerical results it is noticed that the new scheme gives accurate approximations. However, the heigh order accurate schemes are applicable only for linear system or nonlinear system with smooth functions.for nonlinear systems with discontinuies $\mathrm{m}$, those schemes introduce spurious oscillations near discoutinuities.the monotone schemes approximate the solution without oscillation and converge to the exact solutions . but the monotone schemes can not be more than first order accurate so mear the discontinuities to over come these difficulties, we add an anti-diffusive term to the scheme . in fact this processes not successful and efficient. But this process is not usually successful and efficient in practice as in theory. [2] introduced the concept and theory of TVD schemes .which can be high order accurate and without oscillations . we applied the TVD techniques [5] and $[6,10]$ to our third order scheme oscillation by using the flux limiters. Also, we extend the scheme to solve nonlinear scalar problems as well as system of nonlinear equation. the new TVD scheme remove the spurious . The paper is consists of seven chap. Give . In section 2, we present the third order scheme and stability properties are proved for initial and initial boundary value problems. The TVD formulation of the new scheme is presented in section 3. In section 4, the extension to nonlinear hyperbolic conservation laws is presented. Numerical tests on the linear and nonlinear equations are performed in section 5 . Numerical results are presented and compared with the exact solutions and other methods. Conclusions are presented in section 7.

\section{NUMERICAL SCHEME}

Consider the hyperbolic conservation law

$$
\begin{array}{ll}
u_{t}+f(u)_{x}=0 & ,-\infty \leq x \leq \infty \quad, t \geq 0 \\
u(x, 0)=u_{0}(x) & , u(0, t)=g(t), t \geq 0
\end{array}
$$

Where $f(u)$ is the physical flux. Firstly, we are going to discuss the linear case $f(u)=a u$, where a is a wave propagation speed. In this paper we use a uniform $\mathrm{x}$-t grid, with mesh size $\mathrm{h}$ in $\mathrm{x}$-direction and $\mathrm{k}$ in $\mathrm{t}$-direction with a fixed ratio $\lambda=\frac{k}{h}$. Let $u_{j}^{n}$ denote the numerical solution at the point $(x, t)=(j h, n k)$ for $j=0,1,2, \ldots, N$ and $\mathrm{n}=0,1,2, \ldots$. To get the approximate solution $\mathrm{u}_{\mathrm{j}}^{\mathrm{n}}$, we assume two step method:

$$
\begin{aligned}
& \tilde{\mathrm{u}}_{\mathrm{j}}^{\mathrm{n}+1}=\frac{1}{2}\left(\mathrm{u}_{\mathrm{j}+1}^{\mathrm{n}}+\mathrm{u}_{\mathrm{j}-1}^{\mathrm{n}}\right)-\frac{\mathrm{c}}{2}\left(\mathrm{u}_{\mathrm{j}+1}^{\mathrm{n}}-\mathrm{u}_{\mathrm{j}-1}^{\mathrm{n}}\right) \\
& \mathrm{u}_{\mathrm{j}}^{\mathrm{n}+2}=\mathrm{u}_{\mathrm{j}}^{\mathrm{n}}-\mathrm{c}\left(\tilde{\mathrm{u}}_{\mathrm{j}+1}^{\mathrm{n}+1}-\tilde{\mathrm{u}}_{\mathrm{j}-1}^{\mathrm{n}+1}\right)
\end{aligned}
$$

The parameter $\mathrm{c}=\lambda \mathrm{a}$ is the Courant number. The (2.2a) step is the diffusion scheme and the second is the Leap frog scheme. The scheme (2.2) can be written in one step method as follows:

$$
\begin{gathered}
u_{j}^{n+2}=u_{j}^{n}-\frac{c}{2}\left(u_{j+2}^{n}-u_{j-2}^{n}\right)+\frac{c^{2}}{2}\left(u_{j+2}^{n}-2 u_{j}^{n}+u_{j-2}^{n}\right) \\
j=2,3, \ldots \ldots . N, \quad n=0,1,2, \ldots \ldots \ldots
\end{gathered}
$$

Using Taylor expansion, we see that a scheme (2.3) is third order in both space and time, i.e., of order $\left(h^{3}+k^{3}\right)$. 


\section{Stability Analysis}

Now, we analyse the propagation of the initial data by taking a single Fourier mode $\mathrm{u}(\mathrm{x}, \mathrm{t})=\mathrm{e}^{\mathrm{i}(\omega \mathrm{t}-\zeta \mathrm{x})}$, where $\omega$ is the frequency and $\zeta$ is the wave number and each mode are transported with unit amplitude at a constant speed $\mathrm{c}=\frac{\omega}{\zeta}$. Now, we discuss the properties of the scheme (2.3). Firstly,by the Von Neumann method for stability where we assume that the finite difference approximation $u_{j}^{n}$ can be written as

$$
\mathrm{u}(\mathrm{x}, \mathrm{t})=\mathrm{g}^{\mathrm{n}} \mathrm{e}^{\mathrm{i} \zeta \mathrm{h}}, \quad \mathrm{x}_{\mathrm{j}}=\mathrm{jh}
$$

Substituting in (2.3) we obtain the amplification factor $\mathrm{g}$ as

$$
|g|^{2}=1-4 c^{2}(\xi h)^{2}\left(1-c^{2}\right)
$$

Therefore, the scheme (2.3) is stable if and only if

$$
|c| \leq 1
$$

We notice from (2.5) that the order of dissipation is four and the accuracy is three, while all the second (and higher) order schemes have at least sixth order of dissipation. Therefore, the scheme (2.3) with the lowest order of dissipation among all second and higher order schemes is very appropriate for solving wave propagation problems. Since the scheme (2.3) uses a five-point lattice. We define the following boundary conditions

$$
\begin{array}{ll}
\mathrm{u}_{1}^{\mathrm{n}}=\frac{1}{2}\left(3 \mathrm{u}_{2}^{\mathrm{n}}-\mathrm{u}_{4}^{\mathrm{n}}\right) & \text { for } \quad \mathrm{c} \geq 0 \\
\mathrm{u}_{0}^{\mathrm{n}} \quad \text { is given } & \\
\mathrm{u}_{1}^{\mathrm{n}}=\frac{1}{2}\left(3 \mathrm{u}_{2}^{\mathrm{n}}-\mathrm{u}_{4}^{\mathrm{n}}\right) & \text { for } \quad \mathrm{c} \leq 0 \\
\mathrm{u}_{0}^{\mathrm{n}}=2 \mathrm{u}_{1}^{\mathrm{n}}-\mathrm{u}_{2}^{\mathrm{n}} &
\end{array}
$$

These boundary conditions are written in the form

$$
\mathrm{u}_{1}^{\mathrm{n}}=\frac{1}{2}\left(3 \mathrm{u}_{2}^{\mathrm{n}}-\mathrm{u}_{4}^{\mathrm{n}}\right) \quad, \mathrm{u}_{\mathrm{N}-1}^{\mathrm{n}}=\frac{1}{2}\left(3 \mathrm{u}_{\mathrm{N}-2}^{\mathrm{n}}-\mathrm{u}_{\mathrm{N}-4}^{\mathrm{n}}\right), \quad \mathrm{u}_{\mathrm{N}}^{\mathrm{n}}=2 \mathrm{u}_{\mathrm{N}-1}^{\mathrm{n}}-\mathrm{u}_{\mathrm{N}-2}^{\mathrm{n}} \quad \mathrm{u}_{0}^{\mathrm{n}} \quad \text { is given }
$$

Now we discuss the stability of the method defined by (2.3) and (2.6) in the sense of GKS [1]. Using the stability definition 3.3 in [1] and theorem 5.4 which showed that the stability of two related quarter-plane problem is equivalent to stability for the two boundary problems. The quarter-plane problems are obtained by removing one or the other of the boundaries and extending the domain appropriately to $\pm \infty$. Equation (2.4) can be written in more convenient form

$u_{j}^{n}=\kappa^{j} z^{n}$

where ${ }^{\mathrm{z}=\mathrm{e}^{\mathrm{i} \omega \mathrm{k}},}, \kappa=\mathrm{e}^{-\mathrm{i} \zeta \mathrm{h}}$. If ${ }^{\omega}$ or $\zeta_{\mathrm{is}}$ real, then $|\mathrm{z}|=1$ or $|\kappa|=1$ respectively, but $\omega$ and $\zeta$ may be complex.

\subsection{GKS Stability Theorem}


Models (2.3) and (2.6) are stable if and only if they admit no Eigen solutions ( $\kappa$ ) with $|z| \geq 1$.

For the right quarter problem (2.3) $\mathrm{b} \leq \mathrm{x} \leq \infty, \mathrm{c}>0$, the characteristic equation associated with scheme (2.3) takes the form

$$
\mathrm{P}_{1}(\kappa)=\left(\frac{\mathrm{c}^{2}}{2}-\frac{\mathrm{c}}{2}\right) \kappa^{4}+\left(1-\mathrm{z}-\mathrm{c}^{2}\right) \kappa^{2}+\left(\frac{\mathrm{c}^{2}}{2}+\frac{\mathrm{c}}{2}\right)=0
$$

\section{Lemma 2.1}

Let $\mathrm{Z}=\mathrm{e}^{\mathrm{i} \omega \mathrm{k},}, \kappa=\mathrm{e}^{-\mathrm{i} \zeta \mathrm{h}} \cdot$ therefore

a) the roots of $P_{1}(\kappa)\{z=1\}$ are given by

$$
q_{1}=1, q_{2}=-1, \quad q_{3}=i \sqrt{\frac{1+c}{1-c}}, q_{4}=-i \sqrt{\frac{1+c}{1-c}}
$$

b) If $|z| \geq 1, z \neq 1$ then the roots of $P_{1}(\kappa)$ is divided into two groups (independent of $z$ ):

$$
\mathrm{M}=\left(\kappa_{1}, \kappa_{2}\right), \mathrm{S}=\left(\kappa_{3}, \kappa_{4}\right) \text {, where }\left|\kappa_{1}(\mathrm{z})\right| \leq 1,\left|\kappa_{2}(\mathrm{z})\right| \leq 1, \quad\left|\kappa_{3}(\mathrm{z})\right| \geq 1 \text { and }\left|\kappa_{3}(\mathrm{z})\right| \geq 1
$$

and the inequalities are taken strictly if $|z|>1$.

c) The two roots $\kappa_{1}, \kappa_{2}$ of part (b) are continuations of the roots $q_{i}($ at $z=1$ )

$$
\begin{array}{ll}
\mathrm{M}=\left(\mathrm{q}_{1}, \mathrm{q}_{2}\right), & 0<\mathrm{c} \leq 1 \\
\mathrm{M}=\left(\mathrm{q}_{3}, \mathrm{q}_{4}\right), & -1<\mathrm{c} \leq 0
\end{array}
$$

\section{Proof}

The part (a) is trivial.

The part (b) follows from ([1]) .

c) From (b) we have that for any $|z| \geq 1, z \neq 1$ only two roots for which $|\kappa| \leq 1$ Firstly, we consider c $>0$. It is easy to see that $\kappa=1$ cannot be a double root at $\mathrm{z}=1$. If we consider a perturbation $\mathrm{z}=1+\delta$ with $\delta>0$, and if $\kappa=1+\varepsilon$, then we get from (2.7) that $\varepsilon=-\frac{\delta}{2 c+2 \delta}<0$. Therefore, the continuation of $\mathrm{q}_{1}$ is in the unit circle for $\delta>0$ and $\mathrm{q}_{1}$ is always in $M$. To see the continuation of the root $\mathrm{q}_{2}=-1$ we take $\mathrm{z}=1+\delta, \kappa=-(1+\varepsilon)$ then we get $\varepsilon=-\frac{\delta}{2 \mathrm{c}+2 \delta}<0$ Therefore the continuation of $\mathrm{q}_{2}$ is inside the unit circle for $\mathrm{c}>0$.

For $c<0$, the perturbation argument observable that if $\mathrm{z}=1+\delta, \kappa=1+\varepsilon \Rightarrow \varepsilon>0$ then $\kappa$ is outside the unit circle for $\delta>0$ and similarly for $\mathrm{q}_{2}$ is outside the unit circle. Therefore, according to GKS theorem, $\left(\mathrm{q}_{3}, \mathrm{q}_{4}\right)$ are inside the unit circle.

\section{Theorem 2.1}

For fixed c, the scheme (2.3) with the boundary formula (2.6) is stable in the sense of GKS theory.

\section{Proof}


Forc $>0$ the general solution of the equation (2.3) is $\tilde{\mathrm{u}}_{\mathrm{j}}=\sigma_{1} \kappa_{1}^{\mathrm{j}}+\sigma_{2} \kappa_{2}^{\mathrm{j}}$ where $\kappa_{1} \neq \kappa_{2}$ are the roots of the characteristic equation $\mathrm{P}_{1}(\kappa)=0$ i.e., $\tilde{\mathrm{u}}_{1}=\sigma_{1} \kappa_{1}+\sigma_{2} \kappa_{2}$ and $\tilde{\mathrm{u}}_{0}=\sigma_{1}+\sigma_{2}$. The characteristic equation associated with the boundary condition (2.6) is given by

$\mathrm{Q}_{1}(\kappa)=\kappa(\kappa-1)^{2}(\kappa+2)$

For the condition (2.6a) we have the boundary equations (see [4]for details)

$\kappa_{1}\left(\kappa_{1}-1\right)^{2}\left(\kappa_{1}+2\right) \sigma_{1}+\kappa_{2}\left(\kappa_{2}-1\right)^{2}\left(\kappa_{2}+2\right) \sigma_{2}=0$

The GKS theorem may be written in another form (see[1])

"The homogeneous equations (2.9) have no nontrivial solutions for $|z| \geq 1$ " i.e.,

$$
\operatorname{det} \mathrm{D}(\mathrm{z}) \neq 0
$$

The system (2.9) has a nontrivial solution only if $\kappa_{1}=\kappa_{2}$ and $\kappa_{1}=\kappa_{2}=1$ and since $\kappa=1$ is not double root the $\mathrm{n} Q$ cannot be vanished. Hence no simple root can satisfy (2.10). For a double root,

$$
\begin{gathered}
\tilde{\mathrm{u}}_{\mathrm{j}}=\left(\sigma_{1}+\mathrm{j} \sigma_{2}\right) \kappa^{\mathrm{j}} \text { we have the system } \\
\cdots \cdots \cdots \cdots \cdot \sigma_{1}=0 \\
\kappa(\kappa-1)^{2}(\kappa+2) \sigma_{1}+\left(4 \kappa^{4}-6 \kappa^{2}+2 \kappa\right) \sigma_{2}=0
\end{gathered}
$$

This has nontrivial solutions if $\kappa=0$ or $\kappa=1$ or $\kappa=\frac{-1 \pm \sqrt{3}}{2}$. As before $\kappa=1$ is can not be a double root of $\mathrm{P}_{1}(\kappa)$ and therefore does not satisfy $(2.10)$ while $\kappa=0$ is the trivial solution. It remains only to verify that $\kappa=\frac{-1 \pm \sqrt{3}}{2}$ is not double root. It is clear that $\kappa=\frac{-1-\sqrt{3}}{2}$ has a modulus greater than one and therefor is not in M. Similarly, the root $\kappa=\frac{-1+\sqrt{3}}{2}$ is not double root.

For $\mathrm{c}<0$, and the boundary equations

$$
\begin{aligned}
& \quad\left(\kappa_{1}-1\right)^{2} \sigma_{1}+\left(\kappa_{2}-1\right)^{2} \sigma_{2}=0 \\
& \kappa_{1}\left(\kappa_{1}-1\right)^{2}\left(\kappa_{1}+2\right) \sigma_{1}+\kappa_{2}\left(\kappa_{2}-1\right)^{2}\left(\kappa_{2}+2\right) \sigma_{2}=0
\end{aligned}
$$

This system has a nontrivial solution only if i) $\kappa_{1}=1$, ii) $\kappa_{1}=-1$ and $\kappa_{2}= \pm 1$, iii) $\kappa_{1}=\kappa_{2}$. We have, from the last lemma, $\kappa=1$ is not in $\mathrm{M}$ for $\mathrm{c}<0$. Therefore no simple root can satisfy (2.10). For a double root, we have the system

$$
\begin{gathered}
(\kappa-1)^{2} \sigma_{1}+\left(4 \kappa^{4}-4 \kappa^{2}\right) \sigma_{2}=0 \\
\kappa(\kappa-1)^{2}(\kappa+2)^{2} \sigma_{1}+4 \kappa^{4}-6 \kappa^{2}+2 \kappa \sigma_{2}=0
\end{gathered}
$$

This has nontrivial solutions if $\kappa=0$ or $\kappa=1$ or $\kappa=-1$. As before the cases $\kappa=1,0$ are is not difficult and -1 is not double root. Therefore, the stability is proved. 


\section{NUMERICAL EXAMPLES}

Firstly, we show the accuracy of the schemes on the linear scalar problems.

\section{Example 1}

We solve the transport equation

$$
\mathrm{u}_{\mathrm{t}}+\mathrm{u}_{\mathrm{x}}=0, \quad \mathrm{x} \in[-1,1]
$$

subjected to periodic initial condition

$$
\mathrm{u}(\mathrm{x}, 0)=\sin (\pi \mathrm{x})
$$

and periodic boundary conditions. We compute the results $t=2$. Table 1 shows the convergence rates and errors in $\mathrm{L}^{1}$ norm of the scheme (2.3) with (2.6) which denoted by Amro and the third order scheme presented in [5] denoted by Toro. We note that both the schemes are third order accurate. We notice that our scheme is more accurate. Moreover, the size errors by Amro are smaller than those by Toro scheme.

Table 1.Convergence rates and errors for example 1 at $\mathrm{t}=2$.

\begin{tabular}{|l|l|l|l|l|}
\hline $\mathrm{N}$ & \multicolumn{1}{|l|}{ TORO } & \multicolumn{1}{l|}{ TORO } & Amro & Amro \\
\hline & $\mathrm{L}^{1}$ error & $\mathrm{L}^{1}$ order & $\mathrm{L}^{1}$ error & $\mathrm{L}^{1}$ order \\
\hline 80 & $3.65 \mathrm{E}-3$ & & $2.72 \mathrm{E}-3$ & \\
160 & $4.65 \mathrm{E}-4$ & 2.97 & $3.06 \mathrm{E}-4$ & 3.15 \\
320 & $5.95 \mathrm{E}-5$ & 2.97 & $3.74 \mathrm{E}-5$ & 3.03 \\
640 & $7.63 \mathrm{E}-6$ & 2.96 & $4.17 \mathrm{E}-6$ & 3.16 \\
\hline
\end{tabular}

\section{TVD VERSION OF THE THIRD ORDER SCHEME}

we will construct the TVD version of the scheme (3.3) and (3.6) presented in the last section. Firstly, we consider the linear hyperbolic conservative law

$$
\mathrm{u}_{\mathrm{t}}+\mathrm{f}(\mathrm{u})_{\mathrm{x}}=0, \quad \mathrm{f}(\mathrm{u})=\mathrm{au},
$$

where a constant. The scheme (2.3) may be written in the conservative form

$$
u_{j}^{n+1}=u_{j}^{n}-\lambda\left[F_{j+1 / 2}^{n}-F_{j-1 / 2}^{n}\right]
$$

here $\mathrm{F}_{\mathrm{j}+\frac{1}{2}}$ is the numerical flux takes the form [5]

$$
\mathrm{F}_{\mathrm{j}+1 / 2}=\frac{1}{2}\left(\mathrm{au}_{\mathrm{j}}+\mathrm{au}_{\mathrm{j}+1}\right)-\frac{1}{2}|\mathrm{a}| \Delta_{\mathrm{j}+1 / 2} \mathrm{u}+|\mathrm{a}|\left\{\mathrm{A}_{0} \Delta_{\mathrm{j}+1 / 2} \mathrm{u}+\mathrm{A}_{1} \Delta_{\mathrm{j}+\mathrm{L}+1 / 2} \mathrm{u}+\mathrm{A}_{2} \Delta_{\mathrm{j}+\mathrm{M}+1 / 2} \mathrm{u}\right\}
$$


where

$$
\mathrm{A}_{0}=\frac{1}{2}-\frac{|\mathrm{c}|}{2}, \quad \mathrm{~A}_{1}=-\left(\frac{1}{4}+\frac{|\mathrm{c}|}{8}\right), \quad \mathrm{A}_{2}=\frac{1}{4}-\frac{|\mathrm{c}|}{4}
$$

$$
\mathrm{L}=-1, \mathrm{M}=1 \quad \text { for } \mathrm{c}>0 \quad \text { and } \quad \mathrm{L}=1, \mathrm{M}=-1 \text { for } \quad \mathrm{c}<0 \text { and } \Delta_{\mathrm{j}+1 / 2} \mathrm{u}=\mathrm{u}_{\mathrm{j}+1}-\mathrm{u}_{\mathrm{j}}
$$

\subsection{TVD form of the method}

The total variation $\mathrm{TV}\left(\mathrm{u}_{\mathrm{j}}^{\mathrm{n}}\right)$ of the mesh function $\mathrm{u}^{\mathrm{n}}$ is defined as

$$
\mathrm{TV}\left(\mathrm{u}^{\mathrm{n}}\right)=\sum_{-\infty}^{\infty}\left|\mathrm{u}_{\mathrm{j}+1}^{\mathrm{n}}-\mathrm{u}_{\mathrm{j}}^{\mathrm{n}}\right|=\sum_{-\infty}^{\infty}\left|\Delta_{\mathrm{j}+1 / 2} \mathrm{u}^{\mathrm{n}}\right|
$$

The scheme (4.2) is said to be TVD scheme if

$$
\mathrm{TV}\left(\mathrm{u}^{\mathrm{n}+1}\right) \leq \mathrm{TV}\left(\mathrm{u}^{\mathrm{n}}\right)
$$

which means that the total variations not increased as time evolves, so that $\mathrm{TV}\left(\mathrm{u}^{\mathrm{n}}\right)$ for any time $\mathrm{n}$ is bounded by $\mathrm{TV}\left(\mathrm{u}^{0}\right)$ of the initial data. If the initial data of equation (4.1) is smooth, then the total variation of the solution remains constant and if the shock is formed the total variation decreases.

To make the scheme TVD, we use Harten's theorem [2], that states that the scheme written as

$$
u_{j}^{n+1}=u_{j}^{n}-B_{j-1 / 2} \Delta_{j-1 / 2} u+C_{j+1 / 2} \Delta_{j+1 / 2} u
$$

is TVD scheme provided that

$$
\mathrm{B}_{\mathrm{j}+1 / 2} \geq 0, \quad \mathrm{C}_{\mathrm{j}+1 / 2} \geq 0 \quad \mathrm{~B}_{\mathrm{j}+1 / 2}+\mathrm{C}_{\mathrm{j}+1 / 2} \leq 1
$$

where $\mathrm{B}_{\mathrm{j}+1 / 2}$ and $\mathrm{C}_{\mathrm{j}+1 / 2}$ are data dependent coefficients i.e., functions of the set $\left\{u_{j}^{n}\right\}$.

Applying the TVD constraint on (4.3) by a flux limiter functions gives

$$
\mathrm{F}_{\mathrm{j}+1 / 2}=\frac{1}{2}\left(\mathrm{au}_{\mathrm{j}}+\mathrm{au}_{\mathrm{j}+1}\right)-\frac{1}{2}|\mathrm{a}| \Delta_{\mathrm{j}+1 / 2} \mathrm{u}+|\mathrm{a}|\left\{\mathrm{A}_{0} \Delta_{\mathrm{j}+1 / 2} \mathrm{u}+\mathrm{A}_{1} \Delta_{\mathrm{j}+\mathrm{L}+1 / 2} \mathrm{u}\right\} \phi_{\mathrm{j}}+|\mathrm{a}| \mathrm{A}_{2} \Delta_{\mathrm{j}+\mathrm{M}+1 / 2} \mathrm{u} \phi_{\mathrm{j}+\mathrm{M}}
$$

where $\phi_{\mathrm{j}}$ and $\phi_{\mathrm{j}+\mathrm{M}}$ are the flux limiter functions.

\section{Theorem 4.1}

Scheme (4.2) and (4.9) is TVD for $|c| \leq 1$ if the limiter $\phi_{\mathrm{j}}$ is determined by

$$
\begin{aligned}
\phi_{j} \leq \frac{(1-|c|) r_{j}}{\eta\left(A_{1} r_{j}+A_{0}-A_{2}\right)} \\
\phi_{j} \leq \frac{1-|c|+\eta A_{2} / r_{j}^{*}}{\eta\left(\mathrm{A}_{1} r_{j}+A_{0}\right)}
\end{aligned}
$$




$$
\begin{gathered}
\phi_{\mathrm{j}} \geq \frac{\mathrm{A}_{2}}{\left(\mathrm{~A}_{1} \mathrm{r}_{\mathrm{j}}+\mathrm{A}_{0}\right) \mathrm{r}_{\mathrm{j}}^{*}} \\
\phi_{j} \geq 0
\end{gathered}
$$

Where the $r_{j}$ is defined by

$$
r_{j}=\frac{\Delta_{j+L+1 / 2} u}{\Delta_{j+1 / 2} u}
$$

and $r_{j}^{*}$ the upwind-downward flow parameter and given by

$$
r_{j}^{*}=\frac{\Delta_{j+L+1 / 2} \mathrm{u}}{\Delta_{j+M+1 / 2} \mathrm{u}}
$$

and $\eta$ is

$$
\eta= \begin{cases}1-|c| & \text { for } 0 \leq|c|<1 / 2 \\ |c| & \text { for } 1 / 2 \leq|c| \leq 1\end{cases}
$$

Proof see $([5,10])$.

Using theorem to the scheme (4.2), (4.9), therefore the flux limiter takes the form

$$
\begin{gathered}
\phi_{j}=\left\{\begin{array}{lc}
\frac{(1-|c|) r_{j}}{\eta\left(A_{1} r_{j}+A_{0}-A_{2}\right)} & \text { for } 0 \leq r_{j} \leq r^{L} \\
\frac{1-|c|+\eta A_{2} \phi_{j+M} / r_{j}^{*}}{\eta\left(A_{1} r_{j}+A_{0}\right)} & \text { for } r^{L} \leq r_{j} \leq r^{R} \\
0 & \text { for } r_{j}>r^{R}
\end{array}\right. \\
\phi_{j+M}= \begin{cases}\eta r_{j+M} & \text { for } r_{j}<0 \\
1 & \text { for } 0 \leq r_{j+M}<0.5 \\
0 & \text { for } r_{j}=0\end{cases}
\end{gathered}
$$

where

$$
\mathrm{r}^{\mathrm{L}}=\frac{\eta\left(\mathrm{A}_{0}-\mathrm{A}_{2}\right)}{1-|\mathrm{c}|-\eta \mathrm{A}_{1}}, \quad \mathrm{r}^{\mathrm{R}}=\frac{1-|\mathrm{c}|-\eta\left(\mathrm{A}_{0}-\mathrm{A}_{2} \phi_{\mathrm{j}+\mathrm{M}} / \mathrm{r}_{\mathrm{j}}^{*}\right)}{\eta \mathrm{A}_{1}}
$$

Therefore, the scheme (4.2), (4.9) is TVD. 


\section{EXTENSIONOF THE SCHEME TO NONLINEAR SCALAR HYPERBOLIC CONSERVATION LAWS}

To extend the scheme (5.1) and (5.9) to nonlinear scalar problems, we consider the nonlinear scalar equation

$$
\mathrm{u}_{\mathrm{t}}+\mathrm{f}(\mathrm{u})_{\mathrm{x}}=0 \text {, }
$$

where the wave speed,

$$
a_{j+\frac{1}{2}}=\left\{\begin{array}{lc}
\frac{\Delta_{j+\frac{1}{2}} \mathrm{f}}{\Delta_{j+\frac{1}{2}} \mathrm{u}} & \Delta_{j+\frac{1}{2}} \mathrm{u} \neq 0 \\
\left.\frac{\partial \mathrm{f}}{\partial \mathrm{u}}\right|_{\mathrm{u}_{j}} & \Delta_{j+\frac{1}{2}} \mathrm{u}=0
\end{array}\right.
$$

Now we redefine the $r_{j}$ in (3.11) as

$$
r_{j}=\frac{\left|a_{j+L+\frac{1}{2}}\right| \Delta_{j+L+1 / 2} u}{\left|a_{j+\frac{1}{2}}\right| \Delta_{j+1 / 2} u}, \quad r_{j}^{*}=\frac{\mid a_{j+L+\frac{1}{2}} \Delta_{j+L+1 / 2} u}{\left|a_{j+M+\frac{1}{2}}\right| \Delta_{j+M+1 / 2} u},
$$

Here $c_{j+\frac{1}{2}}=\frac{\Delta t}{\Delta x} a_{j+\frac{1}{2}}$. Unlike the constant coefficient case, $a_{j+\frac{1}{2}}$ and $a_{j-\frac{1}{2}}$. Then the numerical flux (4.9) takes the form

$$
\begin{aligned}
& \mathrm{F}_{\mathrm{j}+1 / 2}=\frac{1}{2}\left(\mathrm{f}_{\mathrm{j}}+\mathrm{f}_{\mathrm{j}+1}\right)-\frac{1}{2}\left|\mathrm{a}_{\mathrm{j}+\frac{1}{2}}\right| \Delta_{\mathrm{j}+1 / 2} \mathrm{u}+\left|\mathrm{a}_{\mathrm{j}+\frac{1}{2}}\right|\left\{\mathrm{A}_{0} \Delta_{\mathrm{j}+1 / 2} \mathrm{u}+\mathrm{A}_{1} \Delta_{\mathrm{j}+\mathrm{L}+1 / 2} \mathrm{u}\right\} \phi_{\mathrm{j}}+ \\
& \left|\mathrm{a}_{\mathrm{j}+\frac{1}{2}}\right|\left\{\mathrm{A}_{2} \Delta_{\mathrm{j}+\mathrm{M}+1 / 2} \mathrm{u}\right\} \phi_{\mathrm{j}+\mathrm{M}}
\end{aligned}
$$

By considering all the possible combinations of the signs of $a_{j+\frac{1}{2}}$ and $a_{j-\frac{1}{2}}$, the sufficient conditions on $\phi$ still have the form similar to (4.14) by replacing ( c) by $\mathrm{a}_{\mathrm{j}+\frac{1}{2}}$.

Remark: For two dimensions scalar equations and for systems of equations we use the same procedure presented in $[8,11]$

\section{NUMERICAL EXPERIMENTS}

In this section, we examine our scheme presented here and compare them with others schemes. In all figures the exact solution is shown by solid lines and the numerical solutionare shown by symbols

\subsection{Scalar problems}

Consider the scalar equation 


$$
\begin{aligned}
& \mathrm{u}_{\mathrm{t}}+\mathrm{u}_{\mathrm{x}}=0, \quad-\infty<\mathrm{x}<\infty, \quad \mathrm{t} \geq 0 \\
& \mathrm{u}(\mathrm{x}, 0)=\mathrm{g}(\mathrm{x})
\end{aligned}
$$

where

$$
\mathrm{g}(\mathrm{x})= \begin{cases}0, & 0 \leq \mathrm{x} \leq 1 \\ \sin [8 \pi(\mathrm{x}-1)] & 1 \leq \mathrm{x} \leq 2 \\ 0 & 2 \leq \mathrm{x} \leq \infty\end{cases}
$$

The numerical solution is displayed with $\mathrm{N}=100$ and $\mathrm{c}=0.9$, at a long-time $\mathrm{t}=9$. Fig 1 and 2 show the results computed by the leap frog scheme and the non TVD scheme (2.3) respectively. The results by the TVD third order scheme (4.2) and (4.9)) is shown in fig 3. By comparing the results with the third order Toro TVD scheme (see fig. 1-a in [11,8]we note the leap frog method introduce spurious oscillations while the non TVD scheme (2.3) reduces these oscillations. Also, Toro scheme is not satisfactory for long time while our TVD scheme gives very good approximations.

\section{2. (Riemann problem)}

We assume the equation (5.1) with the initial condition

$$
g(x)= \begin{cases}1 & 1 / 3 \leq x \leq 2 / 3 \\ 0 & \text { elsewhere }\end{cases}
$$

This equation has travelling discontinuity. We compute the numerical solution at $\mathrm{t}=0.2$, with $\mathrm{N}=180$ and $\mathrm{c}=$ 0.9. Figures 4,5,6 show the results computed by the leap frog, Amro and the TVD scheme (4.2) with (4.9) respectively. We notice that, the leap frog scheme introduces more oscillations as expected while the second method reduces them and the TVD third order scheme (4.2) with (4.9) gives the best approximation.

\subsection{Burgers' equation}

Here we consider the numerical solution of the nonlinear Burgers' equation

$$
u_{t}+\left(\frac{u^{2}}{2}\right)_{x}=0
$$

with initial condition

$$
u(x, 0)= \begin{cases}-1 & |x| \geq 0.5 \\ 2 & |x|<0.5\end{cases}
$$

This problem allow shock - at $\mathrm{x}=0.5$, the jump produces an expansion fan with a sonic point . while the shock wave is created at $x=0.5$ and its speed equals to $(0.5)$. the shock hits the rarefaction at time $t=2 / 3$ and therefor the solution has a rarefaction only. the problem is solved numerically at $t=0.4$ i.e., before the collision of shock and rarefaction and at $\mathrm{t}=1$ i.e. after the collision. The numerical solution are shown in figures 7 a and $b$, with 80 grid points, obtained with our TVD scheme at $t=0.4$ and $t=1$ respectively. We notice that results are very accurate. 


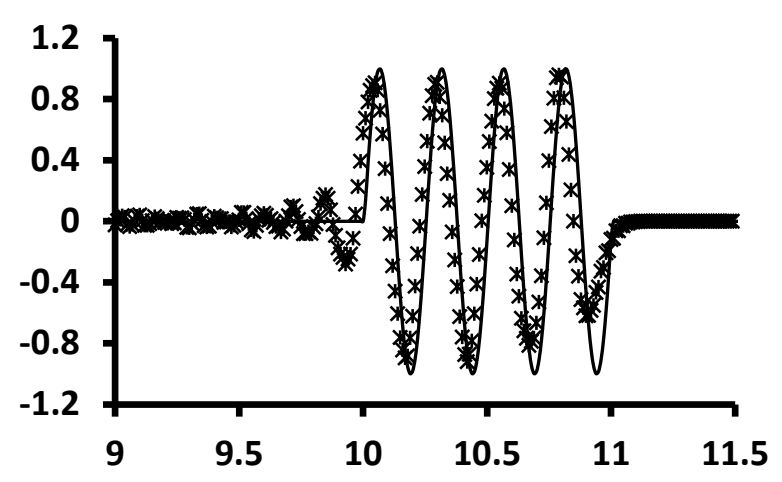

Fig. 1. Solution of problem $(6.1,2)$ using Leap Frog

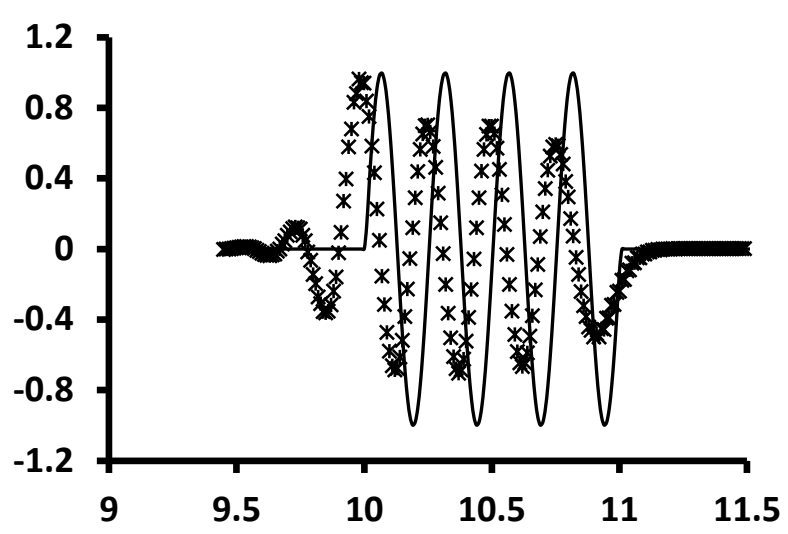

Fig. 2. Solution of problem $(6.1,2)$ using Non-TVD.

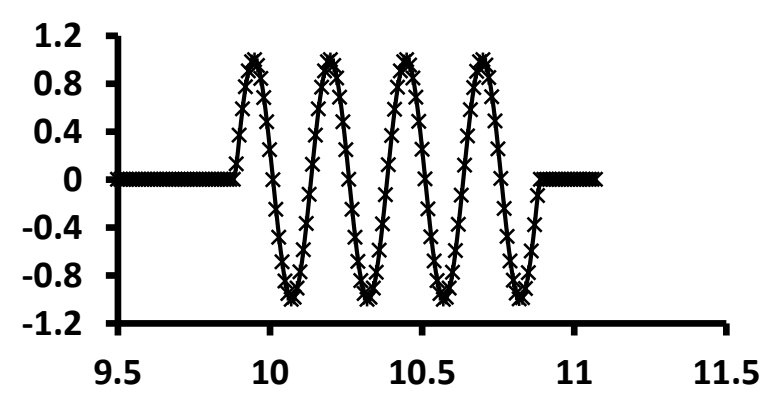

Fig. 3. Solution of the problem $(6.1,2)$ using TVD.

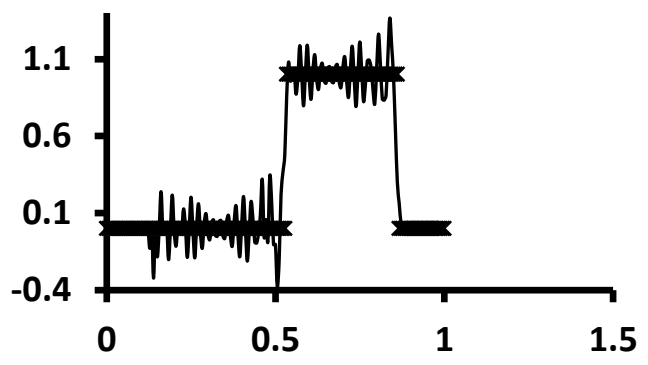

Fig. 4. Solution of (Riemann problem) using Leap frog. 


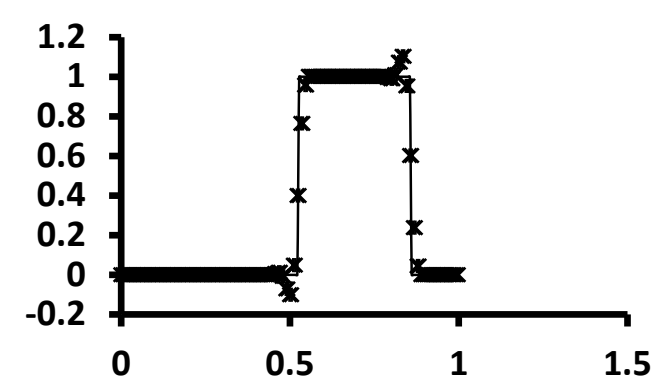

Fig. 5. Solution of (Riemann problem) using Amro.

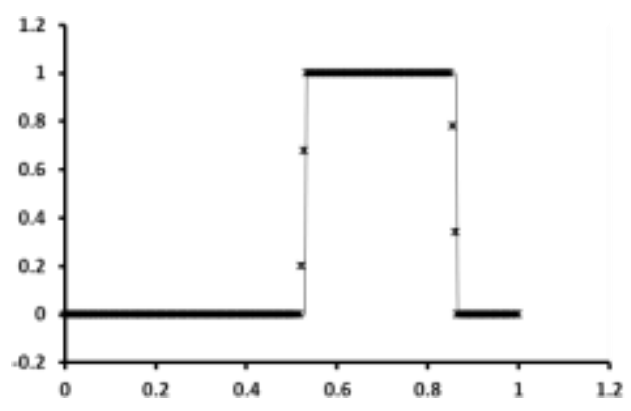

Fig. 6. Solution of (Riemann problem) using TVD.

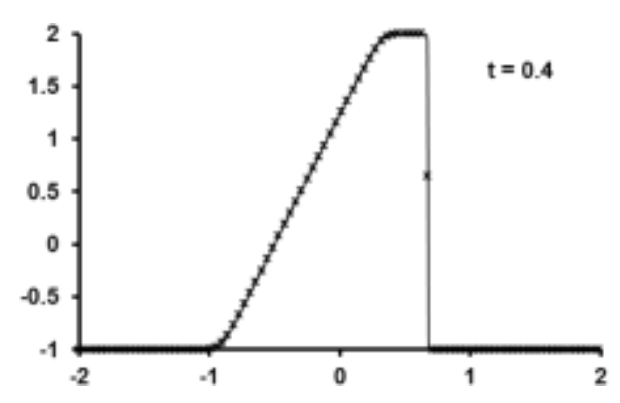

Fig.7a. Solution of problem(6.4) at $t=0.4$

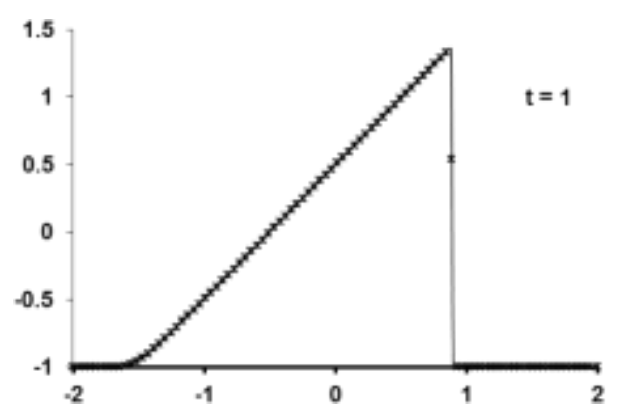

Fig.7b. Solution of (6.4) at $\mathrm{t}=1$.

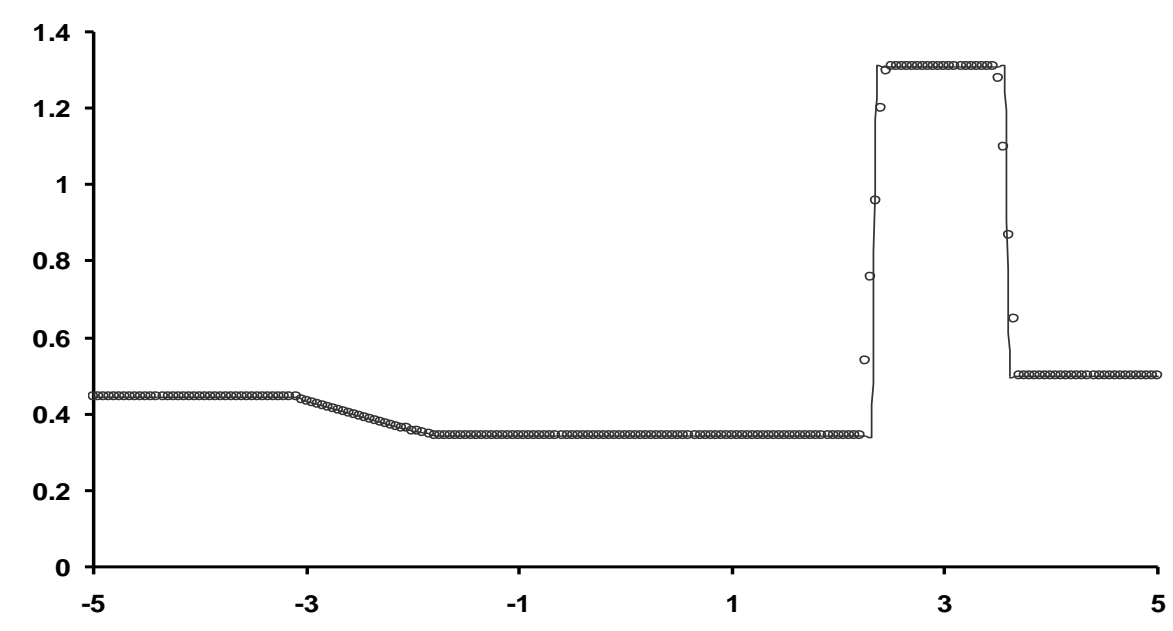

Fig. 8. Solution of Lax problem at $\mathrm{t}=1.3$ with 200 cells. 


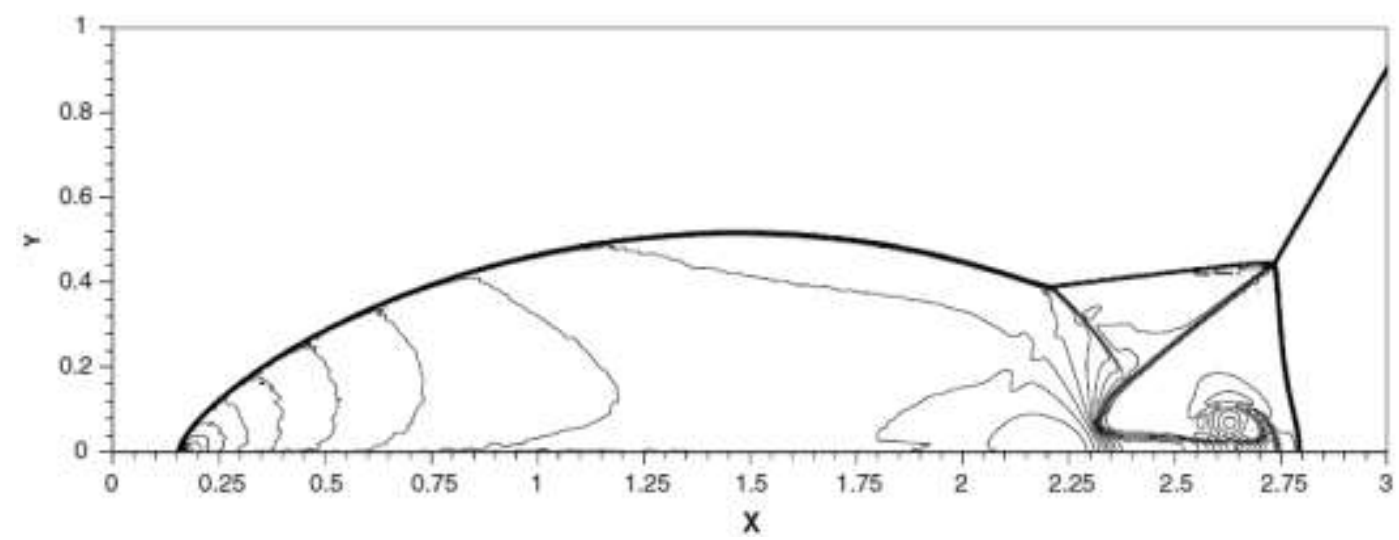

Fig. 9. Solution of the double Mach reflection problem on mesh of 960 x 240.

\subsection{One dimensional system of Euler equations}

We now apply our new scheme to the one-dimensional system Euler equations of gas dynamics

$$
\mathrm{U}_{\mathrm{t}}+\mathrm{F}(\mathrm{U})_{\mathrm{x}}=0
$$

where $U=(\rho, \rho u, E)^{T}$ and $F(U)=\left(\rho u, \rho u^{2}+P, u(E+P)\right)^{T}$. Where $\rho$ is the density, $u$ is the velocity, $\mathrm{P}$ is the pressure, $\mathrm{E}=\frac{1}{2} \rho \mathrm{u}^{2}+\frac{\mathrm{P}}{(\gamma-1)}$ is the total energy and $\gamma$ is the ratio of specific heats, taken as 1.4 here.

\subsubsection{Lax problem}

We solve the Lax problem for Euler equations (5.5) and initial data consists of two states, left (L) and right $(\mathrm{R})$

$$
\left(\rho_{L}, u_{L}, P_{L}\right)=(0.445,0.698,3.528) \quad \text { and } \quad\left(\rho_{R}, u_{R}, P_{R}\right)=(0.5,0.0,0.571)
$$

separated by a discontinuity at $\mathrm{x}=0.0$. The computational domain is taken as the interval $[-5,5]$. Fig. 8 shows the results computed by our TVD scheme at $\mathrm{t}=1.3$ with 200 cells. Comparing with the results obtained with CWENO scheme (Shi J. and Toro E.F., (1996) which generated non-physical oscillations near discontinuities, especially near contact discontinuity while the results obtained by our scheme are oscillations free and is more accurate.

\subsection{Extension to two dimensional problems}

Our scheme can be applied to multidimensional problems. As an example, we consider the two dimensional, Euler equations

$$
\mathrm{U}_{\mathrm{t}}+[\mathrm{F}(\mathrm{U})]_{\mathrm{x}}+[\mathrm{G}(\mathrm{U})]_{\mathrm{y}}=0
$$

where

$$
\mathrm{U}=(\rho, \rho \mathrm{u}, \rho \mathrm{v}, \mathrm{E})^{\mathrm{T}}, \quad \mathrm{F}(\mathrm{U})=\left(\rho \mathrm{u}, \mathrm{P}+\rho \mathrm{u}^{2}, \rho \mathrm{uv}, \mathrm{u}(\mathrm{P}+\mathrm{E})\right)^{\mathrm{T}}, \mathrm{G}(\mathrm{U})=\left(\rho \mathrm{v}, \rho \mathrm{uv}, \mathrm{P}+\rho v^{2}, \mathrm{v}(\mathrm{P}+\mathrm{E})\right)^{\mathrm{T}}
$$

\subsubsection{Double Mach reflection problem}


This equation for this problem is the two-dimensional Euler equations (6.6) presented in[9]. Fig. 9 shows the computed density by our TVD scheme with $960 \times 240$ cells. We note that the scheme produces the flow pattern generally accepted in the present literature[9] as correct. All discontinuities are well resolved and correctly positioned.

\section{CONCLUSIONS}

In this paper, we introduce a third order TVD finite difference scheme for solving conservation laws.the advantages of are: simplicity, high odder accuracy and has the minimum order of dissipation. Many numerical experiments, one- and two-dimensional scalar and system cases are considered and the proposed scheme exhibits excellent performances in all cases.

Applications of the scheme to one and two dimensions for scalar equations and nonlinear systems give results that compare very well with those obtained by existing high resolution methods.

\section{REFERENCES}

[1] Gustafsson, B. H.O. Kriess and A. Sunstrom, “ Approximations for initial boundary value problems II" J. Math.Comp., 26 pp. 649-686, 1972.

[2] Harten, A., "High resolution schemes for hyperbolic conservation laws",J. Comput. Phys. 49pp 357393, 1983.

[3] Roe, P.L) "Approximate Riemann solvers parameter vectors and difference schemes", J.Comput. Phys. 43pp 357-372, 1981.

[4] Turckel, E. "Composite methods for hyperbolic equations" SIAM J. Num. Anal.114744-759, 1977.

[5] Shi J. and Toro E.F., " Fully discrete high order shock capturing numerical schemes", Int.J.Numer.Methods fluids, 23pp 241-269, 1996.

[6] Sweby, P.K., "High resolution schemes using flux limiters for hyperbolic conservation laws", SIAM, J.Num.Anal.21pp 995-1011, 1984.

[7] Trefethen, L.N., "Group velocity in finite difference schemes", SIAM J. Review, 24pp 113-135, 1982.

[8] Vila ,J.P., “ An analysis of class of second order accurate Godunov type schemes”, SIAM J. Num.Anal. 26830-853, 1989.

[9] Woodward, P., and Colella, P.," The numerical solution of two-dimensional fluid flow with strong waves" J.Comput.Phys. 54 pp 115-173, 1984.

[10] Yousef H. Zahran "A Family of TVD second order schemes of nonlinear scalar conservation laws" Comptes rendus de l' Acad. Bulgare des Sci. V 56 April No. 4 pp. 15-22, 2003.

[11] Yousef H. Zahran, "Third order TVD scheme for hyperbolic conservation laws" Bull. Belg. Math. Soc. Simon Stevin 14, 259-275, 2007. 